\title{
From Gas to Stars: Simulating a Population of GMCs
}

\author{
Nathan J. Goldbaum and Mark R. Krumholz \\ Department of Astronomy \& Astrophysics, University of California, Santa Cruz, CA, 95064 \\ email: goldbaum@ucolick.org
}

\begin{abstract}
Here, we present a theoretical model for the global evolution of isolated giant molecular clouds. Accounting for the amount of mass, momentum, and energy supplied by accretion and star formation feedback, we are able to follow the growth, evolution, and dispersal of individual GMCs. We compare our models to observations of GMCs and associated young star clusters in the Large Magellanic Cloud and find good agreement between our model clouds and the observed relationship between H II regions, young star clusters, and GMCs.
\end{abstract}

Keywords. ISM: clouds - ISM: molecules — ISM: evolution — stars: formation — turbulence

\section{Introduction}

Giant molecular clouds (GMCs) are the primary reservoir of molecular gas in the Milky Way (Williams \& McKee 1997, Roman-Duval et al. 2010) and other nearby galaxies (Fukui et al. 2008) (although see Wong et al. (2011), who find that this might not be the case in the Large Magellanic Cloud). Since the surface density of star formation shows a strong correlation with the surface density of molecular gas (Bigiel et al. 2008, Schruba et al. 2011), GMCs must therefore also be the primary site of star formation in these systems.

Understanding how GMCs form, grow, evolve, and eventually disperse is therefore key to understanding how star formation proceeds in quiescent galactic disks. A detailed theoretical model of GMC evolution will aid in interpreting observations of GMCs, which only show a snapshot in the evolution of a cloud, and allow simulations of galaxy formation to capture the observed fact that star formation occurs in dense molecular gas.

What are the most important drivers of molecular cloud evolution? Molecular clouds are roughly virialized, indicating that self-gravity roughly balances turbulent support on the scale of a cloud as a whole. Individual massive star formation events launch H II regions, which can buffet and even destroy a cloud as a while (Murray 2010, Lopez et al. 2011). Lastly, although there is only one direct observation of this process (Fukui et al. 2009), molecular clouds must continuously accrete material (Klessen \& Hennebelle 2010) since the typical velocities and surface density of atomic gas in galaxies are not sufficient to assemble a massive molecular cloud on a timescale much shorter than a typical GMC lifetime ( 230 Myr) (Fukui et al. 2009).

Below, we briefly describe such a model, present the model outputs, and show the results of a detailed comparison between our model and multiwavelength observations of molecular clouds and star formation in the Large Magellanic Cloud.

\section{The GMC Model}

The GMC evolution model solves for the time-evolution of the global properties of model molecular clouds. Employing simplifying assumptions, we derive a set of coupled 
ordinary differential equations that govern the time evolution of the cloud's mass, radius, and velocity dispersion. In contrast with previous work, we follow the flow of gas as it condenses out of the diffuse gas in the envelope surrounding the GMC and falls onto the cloud. Combining the governing evolution equations with a set of initial conditions, model parameters, and a model for the time dependence of the mass accretion rate based on the gravitational collapse of the GMC envelope, we can solve for the time evolution of the cloud.

For a full description of the model, see Goldbaum et al. (2011). A schematic representation of the components of our model is presented in Figure 1. In brief, we employ a virial analysis to derive coupled ordinary differential equations for the evolution of the mass, radius, and velocity dispersion of a cloud. We assume the molecular gas inefficiently forms stars, converting $\sim 1 \%$ of the mass of the cloud into stars per free-fall time (Krumholz et al. 2012). The stars in turn launch H II regions which in turn eject gas in the form of a wind traveling away from the cloud at $\sim 10 \mathrm{~km} \mathrm{~s}^{-1}$. Finally, reservoir material continuously falls out of the reservoir onto the cloud, increasing the cloud mass.

\section{Results}

Here we present outputs from our model, assuming an ambient surface density of $16 M_{\odot} \mathrm{pc}^{-2}$, typical of spiral arm regions. The time evolution of a subsample of 40 simulations are plotted in Figure 2. Clouds accrete mass until $10^{6} M_{\odot}$ of gas has been processed through the accretion flow, after which the accretion rate is instantaneously set to zero, producing the sharp feature at $t \approx 35 \mathrm{Myr}$. The clouds attain a quasi-equilibrium configuration in which mass accretion is roughly balanced by mass ejection. Clouds avoid global collapse by extracting energy from the expansion of $\mathrm{H}$ II regions.

While some clouds are still destroyed by energetic H II regions early in their evolution, over $90 \%$ of these clouds were able to accrete their entire reservoir after $25 \mathrm{Myr}$. At this point, the clouds are generally quite massive, $\sim 1.5 \times 10^{6} M_{\odot}$. Once accretion is shut off, the clouds are no longer confined by accretion ram pressure and lose a portion of the power that had been driving turbulence. For this reason, the velocity dispersion decreases in response to the loss of accretion driven turbulence, and the cloud radius expands in response to the loss of the confining pressure provided by accretion. Before the cloud can dissociate, it attains pressure balance with the ambient ISM at a lower

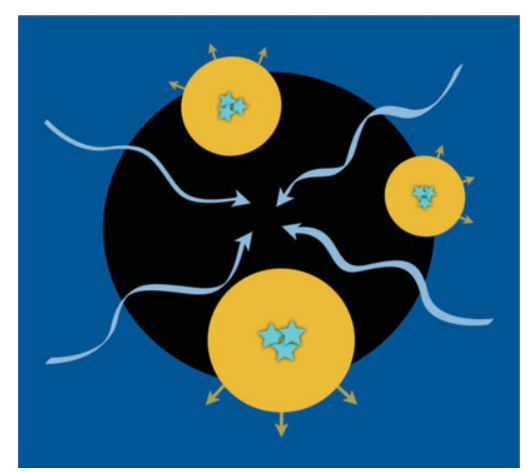

Figure 1. A schematic overview of the GMC model. A molecular cloud is embedded in a warm atomic envelope. Cool atomic gas flows onto the cloud, where it differentially mixes, cools, and adds mass to the molecular cloud. Newborn OB associations drive $\mathrm{H}$ II regions and eject winds ionized winds back into the ambient medium. Image from Goldbaum et al. (2011); Reproduced by permission of the AAS. 
velocity dispersion and larger radius. For the next $20-30 \mathrm{Myr}$, the clouds evolve in much the same way as the massive cloud models considered in Paper I. The clouds can be supported against self-gravity for many dynamical times by forming stars and launching $\mathrm{H}$ II regions. Particularly energetic H II regions can disrupt the clouds and excursions to low surface density can dissociate the clouds. The lifetime of these clouds is thus set by the amount of time they can accrete. This may imply that spiral arm passage times set GMC lifetimes, although further work is needed to clarify this tentative conclusion.

\section{Comparison with Observations}

The Large Magellanic Cloud is home to one of the best-studied samples of GMCs in any galaxy. A large quantity of high-quality multiwavelength data has been obtained for the entire disk of the galaxy. In particular, the NANTEN ${ }^{12} \mathrm{CO}(J=1 \rightarrow 0)$ surveys have mapped the molecular content of the entire disk of the LMC and identified 272 clouds that together contain $5 \times 10^{7} M_{\odot}$ of molecular gas. When combined with multiwavelength archival observations of star formation indicators, these CO data constitute a snapshot in the evolution and star formation history of a population of GMCs.

Kawamura et al. (2009) used the NANTEN CO $J=(1 \rightarrow 0)$ data, along with complementary $\mathrm{H} \alpha$ photometry, radio continuum maps, and a map of young clusters extracted to investigate the ongoing star formation within GMCs in the LMC. These authors found a strong tendency for $\mathrm{H}$ II regions and young clusters to be spatially correlated with GMCs. Using this association, the GMCs in their sample were separated into three types. Type 1 GMCs are defined to be starless in the sense that they are not associated with detectable H II regions or young clusters, Type 2 GMCs are associated with H II regions, but not young clusters in the cluster catalog, and Type 3 GMCs are associated with both $\mathrm{H}$ II regions and young clusters. $24 \%$ of the NANTEN sample were classified as Type 1 , $50 \%$ as Type 2 , and $26 \%$ as Type 3 .

In Figure 3, we show a detailed comparison to this observation. As described in Goldbaum et al. (2011), model GMCs are separated into the classification bins of Kawamura et al. 2009 by calculating the ionizing and bolometric luminosity of the model $\mathrm{H}$ II regions and applying an extinction correction. Using 2000 cloud models, we perform simulated observations using a Monte Carlo scheme. Since low-mass GMCs are far more numerous than massive GMCs, we correct for this by weighting model clouds according to the observed GMC mass function in the LMC.

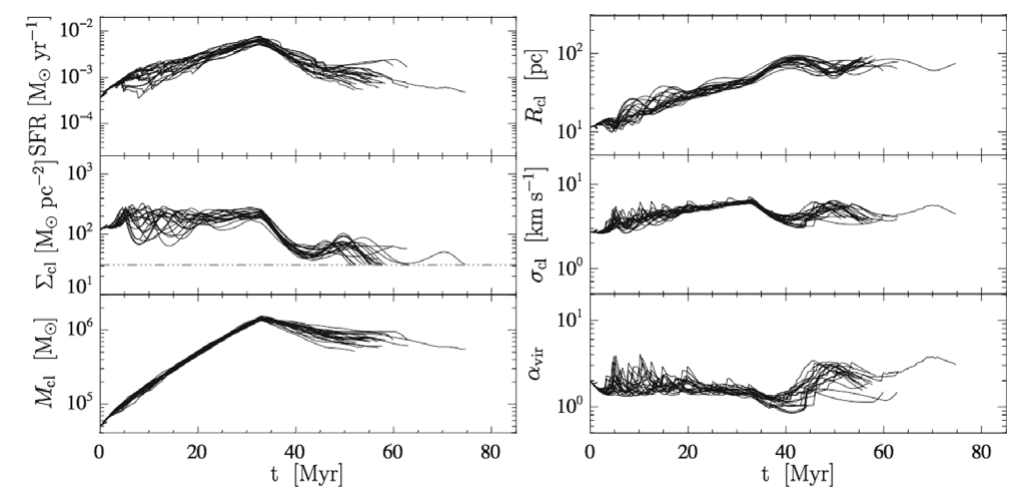

Figure 2. Cloud masses, surface densities, star formation rates, virial parameters, velocity dispersions, and radii for a set of 40 clouds models. Image adapted from Goldbaum et al. (2011); Reproduced by permission of the AAS. 


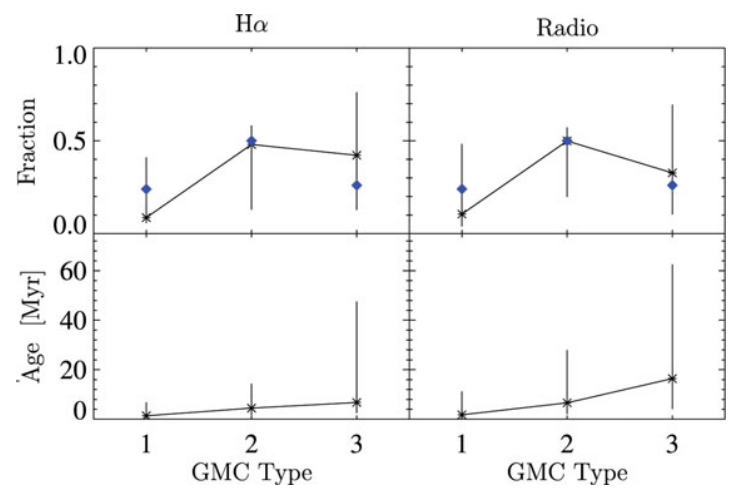

Figure 3. Fraction of GMC lifetime spent as a Type 1, 2, and 3 GMC as defined by Kawamura et al. (2009) (diamonds, top row), and the average age of GMCs in each classification bin (bottom row). The left column identifies Type 2 GMCs via the $\mathrm{H} \alpha$ luminosity, while the right column identifies Type 2 GMCs via radio continuum flux. The error bars encompass the 10th to 90th percentile interval of the probability distribution functions. Image from Goldbaum et al. (2011); Reproduced by permission of the AAS.

The results of this comparison are presented in the top row of Figure 3. In the figure, the lines connect the median of the Monte Carlo probability distributions while the error bars encompass the 10th and 90th percentile. We are able to reproduce the observed distribution of Type 1,2 and 3 GMCs as observed by Kawamura et al. 2009. In particular, using both detection limits, we find that most clouds tend to be Type 2 GMCs, while fewer clouds are Type 1 and 3 GMCs. Interestingly, in the bottom panel of the figure, we find that, on average, the GMC classification scheme does constitute an age sequence in that Type 2 GMCs tend to be somewhat older than Type 1 clouds. Type 3 GMCs in turn tend to be older than Type 2 clouds. On the other hand, the spread in cloud ages within each bin is well within the error bars, indicating that the GMC type classification is not necessarily a strict evolutionary sequence: older clouds can be classified as Type 1 and younger clouds can be classified as Type 3 .

\section{References}

Bigiel, F., Leroy, A., Walter, F., Brinks, E., de Blok, W. J. G., Madore, B., \& Thornley, M. D. 2008, Astronomical Journal, 136, 2846

Blitz, L., Fukui, Y., Kawamura, A., Leroy, A., Mizuno, N., \& Rosolowsky, E. 2007, Protostars and Planets $V, 81$

Fukui, Y., et al. 2008, Astrophysical Journal Supplement, 178, 56

Fukui, Y., et al. 2009, Astrophysical Journal, 705, 144

Fukui, Y. \& Kawamura, A. 2010, Annual Reviews of Astronomy \& Astrophysics, 48, 547

Goldbaum, N. J., Krumholz, M. R., Matzner, C. D., \& McKee, C. F. 2011, Astrophysical Journal, 738,101

Kawamura, A., et al. 2009, Astrophysical Journal Supplement, 184, 1

Klessen, R. S. \& Hennebelle, P. 2010, Astronomy \&3 Astrophysics, 520, A17

Krumholz, M. R., Dekel, A., \& McKee, C. F. 2012, Astrophysical Journal, 745, 69

Lopez, L. A., Krumholz, M. R., Bolatto, A. D., Prochaska, J. X., \& Ramirez-Ruiz, E. 2011, Astrophysical Journal, 731, 91

Murray, N. 2010, arXiv:1007.3270

Roman-Duval, J., Jackson, J. M., Heyer, M., Rathborne, J., \& Simon, R. 2010, Astrophysical Journal, 723, 492

Schruba, A., Leroy, A. K., Walter, F., et al. 2011, Astronomical Journal, 142, 37

Williams, J. P. \& McKee, C. F. 1997, Astrophysical Journal, 476, 166

Wong, T., Hughes, A., Ott, J., et al. 2011, Astrophysical Journal Supplement, 197, 16 